\title{
Evaluation of the inflammatory potential of implant materials in a mouse model by bioluminescent imaging of intravenously injected bone marrow cells
}

Short title: Bioluminescent imaging of inflammation

Bushra Rais ${ }^{1}$, Mario Köster ${ }^{1}$, Muhammad Imran Rahim ${ }^{1}$, Marina Pils ${ }^{1}$, Jan-Marten Seitz ${ }^{2,3}$, Hansjörg Hauser ${ }^{1}$, Dagmar Wirth ${ }^{1}$ and Peter P. Mueller ${ }^{1 *}$

\footnotetext{
${ }^{1}$ Helmholtz Centre for Infection Research, Inhoffenstrasse 7, 38124 Braunschweig, Germany

${ }^{2}$ Institute of Material Sciences, Leibniz University Hannover, An der Universität 2, 30823 Garbsen, Germany
}

${ }^{3}$ Department of Materials Science and Engineering, Michigan Technological University, 1400 Townsend Drive, Houghton, Mi 49931, USA

*Correspondence to: P. P. Mueller; email: pmu@helmholtz-hzi.de

\begin{abstract}
To evaluate the inflammatory potential of implants a bioluminescent imaging assay was developed using luciferase-expressing bone marrow cells that were injected into the blood circulation of wild-type mice. After subcutaneous implantation of titanium discs as an example for a clinically established biocompatible material, the luminosity was modest. Similarly, low luminosity signals were generated by pure magnesium implants that were used to represent metallic alloys that are presently under investigation as novel degradable implant materials. Increased luminosity was observed in response to degradable polymeric PLGA implants. Surgical wounds induced a basic luminescent response even in the absence of an implant. However, the material-independent response to injury could be minimized using injectable microparticle suspensions. In parallel with the resorption of biodegradable
\end{abstract}


microparticles, the signal induced by PLGA declined faster when compared to the stable polymer polystyrene. By using an interferon type I inducible Mx2 promoter construct to drive luciferase gene expression, the highest luminosity was observed in response to bacteria, indicating that the system could also be employed to monitor implant infections. Overall, labeled bone marrow cells yielded specific, well-defined localized signals that correlated with the inflammatory responses to implants.

Keywords: Bioluminescent imaging; bone marrow cells; implant material; inflammation; mouse model 


\section{Introduction}

With the increasing age of the human population, there is a growing demand for medical implants. Many of the presently used materials used for manufacturing implants have originally been developed for technical applications and could be improved to advance their functionality or to patient-specifically customize the performance ${ }^{1}$. Implant materials have been shown to stimulate inflammation to various degrees. Clinically established titanium alloy bone implants have been shown to be highly biocompatible ${ }^{2}$. Polymeric microparticles can be used for diagnostics or therapeutic applications ${ }^{3,4}$. Even though the inflammatory potential is higher, the clinically established degradable poly (lactic-co-glycolic acid) (PLGA) or the stable polystyrene are investigated as particles for drug delivery or for imaging purposes $\frac{4-10}{}$. Chitosan is used in wound dressings and for experimental drug delivery ${ }^{11,12}$. The inflammatory potential of chitosan depends on several factors such as the anatomic site of application and the degree of purity $\underline{13}$. As an example for novel materials still under investigation, magnesium alloys have been proposed as a sturdy biodegradable implant materials for temporary applications ${ }^{14}$. In cell culture assays controversial results concerning the cell compatibility were reported, depending on the specific assay used ${ }^{15-18}$. In animal models magnesium alloys were found to be biocompatible, similar to the titanium alloys ${ }^{19}$.

Minimizing the inflammation in response to implant materials is thought to be advantageous to avoid side effects like delayed wound healing, excess fibrosis or in stent stenosis. Therefore, tissue responses to novel implant materials are evaluated in experimental models before considering clinical applications. To biologically characterize novel implant materials two-dimensional cell culture tests are most frequently used as a primary evaluation method for cell-material interactions ${ }^{20-22}$. However, interactions in vivo are much more complex due to temporal coordinated, three-dimensional dynamic interactions of a wide variety of cell types. Even before implantation surgical injuries initiate inflammatory reactions. After implantation 
the implant material surface is quickly covered by components of the blood or of other body fluids. The first inflammatory cells to arrive at the site of implantation are neutrophils from the blood circulation. Neutrophils can secrete inflammatory mediators which attract additional immune cells such as macrophages. Neutrophils can also secrete enzymes such as myeloperoxidase and produce oxygen radicals that can damage pathogens, tissue or sensitive implant materials. Inflammatory responses are necessary for wound healing but excessive inflammatory processes can have undesirable consequences such as excessive fibrosis or even cancer $\frac{23-25}{2}$. Therefore, it is important to investigate inflammatory properties in vivo. Conventionally, histology is used to evaluate the degree of inflammation. However, histology requires animals to be sacrificed for each time point investigated. This can be avoided by in vivo imaging techniques that allow non-invasive monitoring of inflammatory processes in individual animals over the time. Several fluorescent probes are commercially available that can be used to image inflammatory molecules ${ }^{5,7,26}$. However, the resulting signals appear dispersed and cannot be used to distinguish multiple clustered implants in the same animal ${ }^{27}$. Moreover, the light absorption and the fluorescence of ordinary tissue limits the detection sensitivity ${ }^{27,28}$.

Bioluminescence could be used to evaluate the inflammatory potential of implant materials. A significant advantage of in vivo imaging is the possibility to observe individual implants over the time which could help to increase the consistency of the results and to minimize the animal usage. The aim of this study was to provide a proof of principle that bioluminescent mouse models could be used to evaluate inflammatory implants. Luminescently labeled cells could be isolated from luciferase-expressing animals ${ }^{29,30}$. Previous investigations showed that in transgenic mice the basal expression of luciferase from an $\mathrm{Mx} 2$ promoter construct was efficient. Moreover, the promoter has been shown to be inducible by type I or type III interferons and could potentially serve to differentiate sterile implant material-induced inflammation from interferon-stimulating bacterial implant infections ${ }^{31-34}$. In a first attempt 
implant-dependent inflammation could not be visualized due to considerable constitutive luciferase background activity in these mice. To avoid this background activity luciferaseexpressing bone marrow cells were isolated and intravenously injected in wild-type mice. In this approach, the light emission at implantation sites depended on the capacity of luminescent immune cells to migrate and accumulate in the tissue in response to inflammatory signals.

\section{Methods}

\section{Isolation of bone marrow cells}

C57BL/6 mice that expressed luciferase from Mx2 promoter (Mx2 luc) were euthanized by $\mathrm{CO}_{2}$ asphyxiation $\frac{31}{1}$. Femur and tibia bones from the hind legs were isolated and washed in a petri plate filled with ice cold RPMI (Rosewell Park Memorial Institute) medium (Sigma, Germany) supplemented with 1\% Penicillin/Streptomycin (Gibco, Germany) and 1\% Glutamine (Gibco, Germany). The bones were submerged in 70\% ethanol for 1 minute and then transferred to ice cold RPMI media. Then the joints were cut off and the bone marrow was flushed out with ice cold RPMI medium using a syringe (Injekt, B. Braun Melsungen AG, Germany) with a 26G hypodermic needle (Sterican, B. Braun Melsungen AG). Cell suspensions were collected in $50 \mathrm{ml}$ Falcon tubes and centrifuged at $1500 \mathrm{rpm}$ for 5 minutes. Supernatants were discarded and cell pellets were resuspended in erythrocyte lysis buffer. ACK lysis buffer ( $1 \mathrm{ml}$ per mouse) was added, followed by incubation at room temperature for 1.5 minutes. Then $10 \mathrm{ml}$ of RPMI medium was added and intact cells were pelleted by centrifugation at $1500 \mathrm{rpm}$ for 5 minutes. The supernatant was discarded and cells were suspended in $10 \mathrm{ml}$ of RPMI media. Cells were filtered through $100 \mu \mathrm{m}$ strainer (BD falcon, Germany) and counted using an automated cell counter (Beckman Coulter, Germany).

\section{Purification and FACS analysis of neutrophil and monocyte-specific cell fractions}


Neutrophil and monocyte-specific cell fractions were purified from total bone marrow cells by MACS (Magnetic Activated Cell Sorting) using the Neutrophil isolation kit mouse and Monocyte isolation kit mouse (Miltenyi Biotech, Germany), respectively, using a QuadroMACS $^{\text {TM }}$ magnetic bead separator. For FACS analysis, cells were treated with Fc blocking antibody for 20 minutes and then stained for 30 minutes using anti-mouse Ly6G linked to V450 (BD Biosciences) at a dilution of 1:400 and anti-mouse Ly6C antibody linked to APC (BD Biosciences) at a dilution of 1:200 in FACS buffer (PBS $+2 \%$ fetal calf serum (FCS, Biovest, Germany). Labelled cells were washed twice with PBS and then fixed following the manufacturer's instructions (Cytofix/cytoper kit; BD Biosciences, Germany). Purified cell suspensions were analyzed with a BD LSR II flow cytometer (BD Biosciences, Germany) using the FlowJo software (TreeStar, Germany).

\section{Handling of bacteria}

Staphylococcus aureus COL (MRSA) bacteria were streaked out for single colonies on a Lysogeny broth medium (LB) agarose plate and incubated over night at $37^{\circ} \mathrm{C}$. The next day liquid LB cultures were inoculated with a single colony and incubated at $37^{\circ} \mathrm{C}$ on a rotary shaker set to $180 \mathrm{rpm}$. The optical density of the culture was measured at $600 \mathrm{~nm}$ using a biophotometer plus 6132 (Eppendorf, Germany). At an OD of 0.1, $1 \mathrm{ml}$ of the bacterial culture was centrifuged for 5 minutes at maximum speed in an Eppendorf centrifuge. The supernatant was discarded and the pellet was suspended in $1 \mathrm{ml}$ of phosphate buffered saline (PBS). To inactivate the bacteria, the suspension was heated to $75^{\circ} \mathrm{C}$ for 15 minutes and then stored on ice until use. $5 \mu$ of the heat inactivated $S$. aureus suspension was used for subcutaneous injection.

\section{In vitro luminescence assay}


$10^{3}$ bone marrow cells isolated from female C57BL/6 Mx2-luciferase mice were kept in a cell culture incubator (Binder, Germany) under standard conditions in individual wells of 96 well plates in RPMI medium supplemented with $10 \%$ heat inactivated FCS, $1 \%$ Penicillin/Streptomycin, 1\% Glutamine and $5 \mathrm{ng} / \mathrm{ml}$ mouse recombinant in the presence of granulocyte macrophage colony stimulating growth factor (GM-CSF). $5 \mu$ of heat inactivated $S$. aureus suspension in PBS was added to the culture. To determine the luminosity, cell cultures in 96 well plates were centrifuged at $1500 \mathrm{rpm}$ for 5 minutes. The supernatant was carefully removed and $100 \mu \mathrm{l}$ of luciferin (P.J.K, Germany) was used to suspend the cell pellet. The luminosity was recorded by using a plate reader (Tristar, Berthold Technologies). To determine the cell number, cells were stained by adding $2 \mu \mathrm{M}$ Calcein AM (Life Science Technologies, Germany) in PBS to the pelleted cells and incubated at $37^{\circ} \mathrm{C}$ for 30 minutes. Cells were gently washed with $100 \mu$ of pre-warmed PBS and then re-suspended in $100 \mu \mathrm{l}$ of PBS. The fluorescence intensity was determined measured in a Tristar plate reader at excitation and emission wavelengths of $485 \mathrm{~nm}$ and $535 \mathrm{~nm}$, respectively.

\section{Implant preparation}

Individual porous glass beads $4 \mathrm{~mm}$ in diameter and with an average pore size of $60 \mu \mathrm{m}$ (VitraPOR, Germany), plain titanium discs of $7 \mathrm{~mm}$ diameter and $1 \mathrm{~mm}$ thick (3di, Germany) and magnesium discs of $5 \mathrm{~mm}$ diameter and $2 \mathrm{~mm}$ thickness were used as implants $\stackrel{35}{\text {. Plain }}$ titanium discs of $7 \mathrm{~mm}$ diameter and $1 \mathrm{~mm}$ thick (3di) were coated with poly-(lactic-coglycolic acid) (Sigma Aldrich, Germany) by dissolving $50 \mathrm{mg}$ of PLGA powder in $1 \mathrm{ml}$ of acetone. The PLGA solution was added drop-wise to titanium discs kept on hot plate set to $60^{\circ} \mathrm{C}$. Coated discs were dried at room temperature overnight. Chitosan suspensions (Sigma Aldrich) were prepared by dissolving $50 \mathrm{mg}$ of chitosan in $50 \mathrm{ml}$ dissolved in $0.1 \%$ acetic 
acid (Sigma Aldrich). For subcutaneous injections $50 \mu \mathrm{l}$ suspensions of $1 \mu \mathrm{m}$ diameter microspheres of either PLGA or polystyrene (Phosphorex, USA) were used.

\section{Implantation procedures}

Female C57BL/6 mice (Harlan Winkelmann laboratories, Germany) were kept under specific pathogen free conditions. Animals were anesthetized by intraperitoneal injection of ketamine $(10 \mathrm{mg} / \mathrm{kg})$ and xylazine $(4 \mathrm{mg} / \mathrm{kg})$. The dorsal side was shaved using an electric shaver (Aesculap, Germany) and wiped with $70 \%$ ethanol. PLGA or polystyrene microspheres $1 \mu \mathrm{m}$ in diameter suspended in $50 \mu \mathrm{l}$ of PBS were used for subcutaneous injections. Subsequently, freshly isolated luciferase-expressing bone marrow cells were injected into the tail vein. For the implantation of discs, a cut of $1 \mathrm{~cm}$ was made in the dorsal skin using micro dissecting scissors (Fine Science tools, Germany). Then a pouch under the skin was created into which titanium or magnesium discs were inserted. The incision was closed by interrupted suturing using polyglactin filaments (6-0, Ethicon, Germany). As a control, the entire surgical procedure was performed without inserting an implant. All animal experiments were done in accordance with the regulations and with the approval of the ethical board of the Lower Saxony State Office for Consumer Protection and Food Safety (LAVES), permission number $33.42502 / 07-10.5$.

\section{Intravenous injection and bioluminescent imaging}

Immediately after implantation, $10^{6}$ luciferase labelled cells in $150 \mu 1$ PBS were injected into the tail vein of anesthetized C57BL/6 mice. At the time points indicated, $150 \mu \mathrm{lof}(30 \mathrm{mg} / \mathrm{ml})$ luciferin solution (Synchem, Germany) was injected intraperitoneally. After 15 minutes, the luminosity was determined using an in vivo imaging system (IVIS 200, Xenogen/Caliper Life Sciences, Germany). Data were analyzed with the Living image software ${ }^{\circledR}$ 4.3.1 (Caliper Life Sciences, Germany). 


\section{Histological analysis}

Mice were euthanized by $\mathrm{CO}_{2}$ asphyxiation and skin tissue samples from the microsphere injection site were excised and fixed with $4 \%$ formaldehyde for 48 hours. Then the samples were dehydrated in a 70\% ethanol/water solution and then embedded in paraffin. $3 \mu \mathrm{m}$ thick tissue sections were prepared and stained with Hematoxylin/Eosin (H\&E) according to standard laboratory procedures. After heat-denaturation, tissue sections were reacted with neutrophil-specific 1:200 diluted rabbit polyclonal anti-myeloperoxidase antibodies (Medac/Thermo Scietific, RB-373-A0) and with macrophage-specific rabbit polyclonal antiIBA-1 antibodies (Synaptic Systems, 234003).

\section{Results}

\section{In vitro characterization of bioluminescent bone marrow cell fractions}

We have previously generated a transgenic mouse carrying an Mx2 promoter-luciferase reporter construct that was inducible by interferon type I and III as well as by interferon inducers such as double-stranded RNA analogs or bacterial components ${ }^{31}$. The high constitutive luciferase activity in these animals did not allow the detection of implant-specific increases in the luminosity. It was therefore attempted to avoid the dominant background luminosity by using wild-type mice as recipients for luciferase expressing immune cells, thereby exploiting capacity of the luminescently labeled immune cells to accumulate at inflammatory locations. For this purpose it was essential to identify and isolate luciferase expressing, migratory competent immune cell populations. To this end total bone marrow cells from transgenic Mx2-luciferase animals as well as neutrophil and monocyte-specific cell surface antigen-expressing cell fractions were purified using magnetic beads. The purity of the cell fractions was evaluated by FACS analysis ${ }^{36,37}$. As expected, the majority of the neutrophil-specific cell fraction, approximately $85 \%$, stained positive for both antigens, Ly6G 
and Ly6C. 14\% were Ly6G negative which was indicative of contaminating monocytes (Fig. 1; A). Of the purified monocyte fraction $91 \%$ of the cells were stained positive for Ly6C and negative for Ly6G (Fig. 1; B). This degree of purity was deemed sufficient to identify population-specific luminosity signals.

The promoter of the Mx2 luciferase gene expression cassette has previously been shown to be activated by bacterial components ${ }^{33,34}$. To determine if the luciferase activity of the isolated bone marrow cells could be stimulated by bacteria, immune cells were cultured with or without bacterial extracts. To monitor the viable cell density, cells were labeled with an intracellular esterase-activated fluorescent stain. All three primary cell populations could be cultured in vitro for at least 72 hours and any of them could therefore be used for an in vivo inflammation detection assay. Unexpectedly, the cells exhibited a considerable constitutive activity and only marginal effects of bacterial extracts on the luminescence of total bone marrow cells and in the neutrophilic fraction could be detected (Fig. 1; C \& D). In all three bone marrow cell populations used there was a gradual decline over the time of the remarkably high initial luciferase activity with respect to the constitutive fluorescence control. Taken together, this could tentatively be explained by an activated status of the $\mathrm{Mx} 2$ promoter of freshly isolated cells that during the subsequent cultivation gradually returns to basal levels. The basal luciferase activity of the monocyte-specific fraction was higher when compared to neutrophils or total bone marrow cells (Fig. 1 E; RLU). In addition, the fluorescence intensity of the monocyte-specific population increased most prominently, suggesting that these cells also had the highest proliferation rate. Therefore, even though there was no clear evidence for further luciferase induction in the presence of bacteria, the monocyte bone marrow fraction appeared most promising for yielding a detectable luminescence signal in vivo. 


\section{Bioluminescent detection of subcutaneously injected luciferase-expressing bone marrow cells}

To determine if the luminosity of luciferase-expressing primary bone marrow cells was sufficient to permit in vivo monitoring, cells were subcutaneously injected. Luminescence from viable cells that remained at the site of injection could be detected showing that it was feasible to monitor these cells in vivo (Fig. 2; A). When the cells were stimulated by the local injection of heat inactivated bacteria, the luminosity of solely the monocyte-specific fraction transiently increased during the first two days and declined to control levels afterwards (Fig. $2 ; \mathrm{A}, \mathrm{B} \& \mathrm{C})$. This was in agreement with the in vitro findings. Interestingly, the luminescence from all cells remained highly localized and could be detected for over to two weeks.

\section{Bioluminescent detection of bacterially-contaminated implants using luciferase- expressing bone marrow cells.}

To evaluate the capacity of luciferase-labelled bone marrow cells to extravasate from the circulation and accumulate at sites of inflammation, various cell populations were intravenously injected into non-luminescent mice carrying subcutaneous implants. Since in the previous experiment the highest luciferase activity was observed from the macrophage fraction in the presence of bacteria, heat-inactivated $S$. aureus bacteria were subcutaneously injected on top of the implants. After one day and thereafter up to 19 days light emission could be detected from the site of implantations and also from surgical injury in the absence of implants when total bone marrow cells of neutrophil-specific bone marrow cells were injected and for up to 9 days when injecting a monocyte-specific bone marrow cell fraction (Fig. 3; A). The signal intensity from all three cell populations appeared somewhat higher in the presence of bacteria. However, only unfractionated bone marrow cells showed a significant increase in the luminosity for one week in response to bacteria but not the purified neutrophilic or macrophage fractions (Fig. 3; B to D). 
Overall, unlike the entire cell populations under stationary conditions, the macrophagespecific migratory cell fraction that was attracted to the site of implantation did not show any advantage. Therefore, for simplicity and to minimize the influence of the in vitro manipulations on the cell differentiation status, total bone marrow cells were used in all subsequent experiments. The results demonstrated that luminescently labelled bone marrow cells injected into the blood circulation could egress and accumulate at sites of implantation, whereby in particular from unfractionated bone marrow cells the luminosity appeared enhanced by inflammatory bacterial remains.

\section{Differential detection of inflammatory implants by using bioluminescent bone marrow cells}

To distinguish the inflammatory response to different implant materials, the clinically established biocompatible material titanium, magnesium as a representative of novel biodegradable magnesium alloys and PLGA with a reported enhanced inflammatory potential were subcutaneously implanted. Then luciferase-labelled bone marrow cells were injected into the tail vein and the resulting light emission was monitored. The highest luminescence signal could be detected at the PLGA implantation site with peak intensities between day 3 and day 5 (Fig. 4; A \& B). The depiction of the basal surgical lesion induced luminosity was less apparent due to high peak luminosities achieved (Fig. 4; A, color scale). The results suggest that luminosity from the implantation site reflects the inflammatory potential of the implants and that labelled bone marrow cells could be used to differentially detect inflammatory implant materials.

\section{Injectable biomaterials avoid surgical injury-induced luminescence signals}

To increase the sensitivity of the inflammation detection assay, it was attempted to reduce bioluminescent signals due to surgical injuries by using injectable microparticle suspensions. 
Three material preparations with inflammatory potential, PLGA or polystyrene microsphere suspensions and chitosan solutions were subcutaneously injected in mice. Then, luciferaseexpressing total bone marrow cells were intravenously injected. Luminescence was detectable at the site of injection after 2 days (Fig. 5; A). Radiance at the site of saline buffer injections was close to background levels confirming the expectation that injection caused minimum injury (Fig. 5; A \& B). The luminescence in response to PLGA microspheres and to chitosan decreased to background levels after 9 and 12 days, respectively (Fig. 5; A \& B). Since PLGA and chitosan are biodegradable the limited light emission period could be due to material resorption. Accordingly, the signal from polystyrene persisted for more than 13 days. Therefore, luminescently labeled bone marrow cells could distinguish persistent from biodegradable microparticle suspensions.

\section{Correlation between bioluminescence intensity and histological signs of inflammation}

To confirm that the luciferase activity from intravenously injected bone marrow cells correlated with implant material-induced inflammation, inflammatory events induced by micro-particles in the peri-implant tissue were analyzed. PLGA microspheres, polystyrene microspheres and PBS were subcutaneously injected and after 2 to 8 days tissue samples from the sites with the injected materials were removed and histologically examined. After injection of a buffer solution typical layers of skin tissue without any signs of inflammation could be observed (Fig. 6; A). After injection of PLGA microbead suspensions the periimplant tissue had a granular appearance, which is typical of inflamed tissue infiltrated by monocytic cells (Fig. 6; B). The area occupied by the microbeads was positively stained with myeloperoxidase-specific antibodies, a neutrophilic granulocyte and monocyte-specific stain indicative of inflammation. In accord with the expectations for a biodegradable material, both, the area occupied by microbeads as well as the area intensively stained for myeloperoxidase decreased over the time (Fig. 6; B, d2 to d8). In the environment of polystyrene microspheres, 
granulation tissue was apparent (Fig. 6; C). As expected for a non-degradable polymer, during the duration of the experiment the area occupied by the polystyrene microbeads remained constant. In line with this observation and with the results of the bioluminescence assay, the appearance of the granulation tissue remained constant. An additional fibrotic cell layer appeared between the smooth muscle layer and the area occupied by the microspheres, which is a typical inflammatory tissue response to encapsulate and insulate foreign materials (Fig. 6; $\mathrm{C}$, black arrow). The tissue was positively stained for myeloperoxidase, in particular at the tissue-microbead interface, indicating the infiltration by granulocytes (Fig. 6; C). In conclusion, in agreement with the bioluminescent bone marrow cell assay results, clear signs of tissue inflammation were evident in response to injected microbeads but not after injection of solely buffer solution, whereby in both assays the response to degradable polymers was transient and correlated with the disappearance of the injected material. 


\section{Discussion}

To provide a proof of principle for the evaluation of the inflammatory potential of implant materials a bioluminescence-based assay was developed. Transgenic animals expressing interferon-inducible luciferase showed a dominating constitutive background activity. The background could be avoided by using non-luminescent mice carrying implants and inject labeled bone marrow cells into the blood circulation. Inflammatory responses could be observed to both, infectious bacteria and polymeric implants while a minor signals were obtained from highly biocompatible titanium controls ${ }^{27}$. In addition, the modest inflammatory response reported for biodegradable magnesium implants could be confirmed ${ }^{38}$. Importantly, the luminosity signal from the bone-marrow cells was focused to the implantation sites. This allowed the comparison of different implants in a single animal which was an advantage in comparison to the largely delocalized signals resulting from previously investigated inflammation-activated fluorescent indicators. The possibility to compare different materials in the same animal over the time avoided individual variations and decisively helped to achieve more consistent results.

In principle, the sensitivity of the assay could be further enhanced by increasing the amount of injected cells several-fold $\stackrel{45}{ }$. However, immune cells and their interactions are finely balanced. Injected cells are in addition to circulating immune cells of the host. Further increases would lead to a more imbalanced physiological situation unless the host cell populations would be reduced by a pre-treatment or by genetic means. The number of luciferase expressing bone marrow cells that were injected per animal was sufficient to reliably permit the early detection of luminosity differences between inflammatory and more biocompatible implants. Because the signal strength appeared sufficient to demonstrate the feasibility of this approach, more elaborate possibilities were not pursued. 
In the absence of inflammatory stimuli mouse blood contains low numbers of neutrophils ${ }^{39}$. For this reason, the more abundant neutrophil cell surface marker expressing bone marrow cells were used instead. However, whereas mature neutrophils have been demonstrated to be short lived, the luminosity at implantation sites from intravenously injected labeled bone marrow neutrophilic cell fractions remained detectable for up to two weeks ${ }^{40,41}$. Neutrophils life time extensions have been reported in response to inflammatory stimuli like LPS, GMCSF or $\mathrm{TNF}^{42,43}$. Therefore, the inflammatory tissue environment at implantation sites could play an important role in the persistent luminosity. In addition, the presence of initially minor fractions of proliferation competent cells such as monocytes could explain the prolonged luminosity. A first attempt to characterize the surviving cells revealed that the amount of cells that could be recovered from implantation sites was not sufficient for analysis (results not shown). Therefore, the cause of the persistent luminosity obtained from bone marrow-derived neutrophilic cell fractions remains to be investigated.

In his study constitutive luciferase expression of freshly isolated bone marrow cells was essential for the detection of inflammatory events by vivo imaging. The macrophage antigen expressing cell fraction showed the highest luminosity in vitro and appeared to be proliferating which could be of advantage for optimal detection sensitivity. However, a lower basal activity could facilitate the detection of infections by decreasing the material-induced signal and increasing the responsiveness to pathogens. In fact, during in vitro cultivation of the bone marrow cells the luciferase activity declined. It is a well-established fact that differentiation-specific gene expression decreases with time when primary cells are cultured in vitro; therefore this effect was not studied in more detail ${ }^{44}$. Instead, to best preserve the bone marrow cell differentiation status and the capacity to respond to inflammation it was attempted to minimize in vitro handling and cell culturing. Even though there were some 
assay-specific differences in the resulting luminosity signals between different bone marrow cell preparations, unfractionated cells required the least manipulation and in the absence of additional constraints appeared preferable to monitor implant responses.

Implant independent signals were observed at superficial, full thickness skin scissions. The distinct signal could be explained by the unobscured light emission from the immune cells located at the exposed injured tissue surfaces. In contrast, more dimmed signals were apparent from subcutaneous surgical wounding or from non-inflammatory implants that were covered by light-absorbing skin layers. An interference with signals from superficial wounds could be avoided by placing the implant into a skin pocket, whereby the skin incision for the implant insertion adjacent to, rather than on top of the final implant position. Alternatively, superficial wounding could be avoided altogether by using injectable material preparations. Despite minimal injury the remarkable luminosity observed after injection of polymeric microparticles indicated a highly inflammatory reaction that was histologically confirmed, demonstrating that the detected luminosity correlated with the inflammatory response to the material. However, this method could be applied solely for evaluating particle suspensions, soluble materials or implant degradation products.

Overall, the approach appeared suitable to identify and monitor local inflammatory responses to implants. The inflammatory potential of polymers or bacterial components could be confirmed. Future investigations are required to detect more subtle differences the inflammatory potential of implants. Further optimization of the luciferase promoter regulation could facilitate the discrimination between material-induced responses, wound healing reactions and microbial infections.

\section{Conclusion}


The feasibility of a primary evaluation of the inflammatory potential of implants by in vivo imaging of adoptively transferred labeled bone marrow cells could be demonstrated. Inflammatory and non-inflammatory materials could be identified and monitored for up to two weeks after implantation. The method could be employed for the primary in vivo screening of novel implant materials, micro-particles or anti-inflammatory implant coatings. Additional challenges still remain to be addressed, such as the sensitive monitoring of inflammation in hard-tissue implants and also the long-term observation of low-level irritations.

\section{Acknowledgements}

This research was funded by collaborative Research Centre grant, SFB599 by the German Research Foundation (DFG). We would like to express our appreciation to Dr. Stefan Lienenklaus for his expert contribution to in vivo imaging and also for his insightful suggestions. Bushra Rais was a member of the graduate school of the Helmholtz Center for Infection Research, Braunschweig, Germany.

\section{References}

1. Ratner $\mathrm{BD}$, Bryant SJ. Biomaterials: where we have been and where we are going. Annu Rev Biomed Eng 2004;6:41-75.

2. Palmquist A, Omar OM, Esposito M, Lausmaa J, Thomsen P. Titanium oral implants: surface characteristics, interface biology and clinical outcome. J R Soc Interface 2010;7 Suppl 5:S51527.

3. Varde NK, Pack DW. Microspheres for controlled release drug delivery. Expert Opin Biol Ther 2004;4(1):35-51.

4. Meyer RA, Sunshine JC, Green JJ. Biomimetic particles as therapeutics. Trends Biotechnol 2015;33(9):514-524.

5. Selvam S, Kundu K, Templeman KL, Murthy N, Garcia AJ. Minimally invasive, longitudinal monitoring of biomaterial-associated inflammation by fluorescence imaging. Biomaterials 2011;32(31):7785-92.

6. Liu WF, Ma M, Bratlie KM, Dang TT, Langer R, Anderson DG. Real-time in vivo detection of biomaterial-induced reactive oxygen species. Biomaterials 2011;32(7):1796-1801. 
7. Bratlie KM, Dang TT, Lyle S, Nahrendorf M, Weissleder R, Langer R, Anderson DG. Rapid Biocompatibility Analysis of Materials via In Vivo Fluorescence Imaging of Mouse Models. PLoS ONE 2010;5(4):e10032.

8. Yang WW, Pierstorff E. Reservoir-based polymer drug delivery systems. J Lab Autom 2012;17(1):50-8.

9. Middleton JC, Tipton AJ. Synthetic biodegradable polymers as orthopedic devices. Biomaterials 2000;21(23):2335-2346.

10. Varde NK, Pack DW. Microspheres for controlled release drug delivery. Expert Opinion on Biological Therapy 2004;4(1):35-51.

11. Jayakumar R, Prabaharan M, Sudheesh Kumar PT, Nair SV, Tamura H. Biomaterials based on chitin and chitosan in wound dressing applications. Biotechnol Adv 2011;29(3):322-37.

12. Patel MP, Patel RR, Patel JK. Chitosan mediated targeted drug delivery system: a review. J Pharm Pharm Sci 2010;13(4):536-57.

13. Rinaudo M. Chitin and chitosan: Properties and applications. Prog Polym Sci 2006;31(7):603632.

14. Chen Y, Xu Z, Smith C, Sankar J. Recent advances on the development of magnesium alloys for biodegradable implants. Acta Biomaterialia 2014;10(11):4561-4573.

15. Zhen Z, Liu X, Huang T, Xi T, Zheng Y. Hemolysis and cytotoxicity mechanisms of biodegradable magnesium and its alloys. Materials Science and Engineering: C 2015;46(0):202-206.

16. Seuss F, Seuss S, Turhan MC, Fabry B, Virtanen S. Corrosion of Mg alloy AZ91D in the presence of living cells. J Biomed Mater Res B Appl Biomater 2011;99(2):276-81.

17. Nguyen TY, Liew CG, Liu H. An in vitro mechanism study on the proliferation and pluripotency of human embryonic stems cells in response to magnesium degradation. PLoS One 2013;8(10):e76547.

18. Witte F, Hort N, Vogt C, Cohen S, Kainer KU, Willumeit R, Feyerabend F. Degradable biomaterials based on magnesium corrosion. Curr Opin Solid State Mater Sci 2008;12(56):63-72.

19. Badar M, Lünsdorf H, Evertz F, Rahim MI, Glasmacher B, Hauser H, Mueller PP. The formation of an organic coat and the release of corrosion microparticles from metallic magnesium implants. Acta Biomater 2013;9(7):7580-7589.

20. Kirkpatrick CJ, Bittinger F, Wagner M, Kohler H, van Kooten TG, Klein CL, Otto M. Current trends in biocompatibility testing. Proc Inst Mech Eng H 1998;212(2):75-84.

21. Sauberlich S, Klee D, Richter EJ, Hocker H, Spiekermann H. Cell culture tests for assessing the tolerance of soft tissue to variously modified titanium surfaces. Clin Oral Implants Res 1999;10(5):379-93.

22. Schade R, Sikiric MD, Lamolle S, Ronold HJ, Lyngstadass SP, Liefeith K, Cuisinier F, FurediMilhofer $\mathrm{H}$. Biomimetic organic-inorganic nanocomposite coatings for titanium implants. In vitro and in vivo biological testing. J Biomed Mater Res A 2010;95(3):691-700. 
23. Franz S, Rammelt S, Scharnweber D, Simon JC. Immune responses to implants - A review of the implications for the design of immunomodulatory biomaterials. Biomaterials 2011;32(28):6692-6709.

24. Gretzer C, Emanuelsson L, Liljensten E, Thomsen P. The inflammatory cell influx and cytokines changes during transition from acute inflammation to fibrous repair around implanted materials. Journal of Biomaterials Science, Polymer Edition 2006;17(6):669-687.

25. Eming SA, Krieg T, Davidson JM. Inflammation in wound repair: Molecular and cellular mechanisms. Journal of Investigative Dermatology 2007;127(3):514-525.

26. Suri S, Lehman SM, Selvam S, Reddie K, Maity S, Murthy N, Garcia AJ. In vivo fluorescence imaging of biomaterial-associated inflammation and infection in a minimally invasive manner. J Biomed Mater Res A 2015;103(1):76-83.

27. Rais B, Rahim M, Lienenklaus S, Weiss S, Tolle C, Seitz J-M, Menzel H, Hauser H, Müller P. Animal Test Models for Implant-Associated Inflammation and Infections. In: Lenarz T, Wriggers P, editors. Biomedical Technology: Springer International Publishing; 2015. p 175187.

28. Monici M. Cell and tissue autofluorescence research and diagnostic applications. Biotechnol Annu Rev 2005;11:227-56.

29. Boxio R, Bossenmeyer-Pourié C, Steinckwich N, Dournon C, Nüße O. Mouse bone marrow contains large numbers of functionally competent neutrophils. Journal of Leukocyte Biology 2004;75(4):604-611.

30. Zhao E, Xu H, Wang L, Kryczek I, Wu K, Hu Y, Wang G, Zou W. Bone marrow and the control of immunity. Cell Mol Immunol 2012;9(1):11-19.

31. Pulverer JE, Rand U, Lienenklaus S, Kugel D, Zietara N, Kochs G, Naumann R, Weiss S, Staeheli $P$, Hauser $H$, Koster $M$. Temporal and spatial resolution of type I and III interferon responses in vivo. J Virol 2010;84(17):8626-38.

32. Asano A, Jin HK, Watanabe T. Mouse Mx2 gene: organization, mRNA expression and the role of the interferon-response promoter in its regulation. Gene 2003;306(0):105-113.

33. Haller $\mathrm{O}$, Staeheli $\mathrm{P}$, Kochs $\mathrm{G}$. Interferon-induced $\mathrm{Mx}$ proteins in antiviral host defense. Biochimie 2007;89(6-7):812-818.

34. Decker T, Muller M, Stockinger S. The yin and yang of type I interferon activity in bacterial infection. Nat Rev Immunol 2005;5(9):675-87.

35. Rahim MI, Eifler R, Rais B, Mueller PP. Alkalization is responsible for antibacterial effects of corroding magnesium. J Biomed Mater Res A 2015.

36. Fleming TJ, Fleming ML, Malek TR. Selective expression of Ly-6G on myeloid lineage cells in mouse bone marrow. RB6-8C5 mAb to granulocyte-differentiation antigen (Gr-1) detects members of the Ly-6 family. J Immunol 1993;151(5):2399-408.

37. Rose S, Misharin A, Perlman H. A novel Ly6C/Ly6G-based strategy to analyze the mouse splenic myeloid compartment. Cytometry. Part A : the journal of the International Society for Analytical Cytology 2012;81(4):343-350. 
38. Saris NE, Mervaala E, Karppanen H, Khawaja JA, Lewenstam A. Magnesium. An update on physiological, clinical and analytical aspects. Clin Chim Acta 2000;294(1-2):1-26.

39. Pruijt JF, Verzaal P, van Os R, de Kruijf EJ, van Schie ML, Mantovani A, Vecchi A, Lindley IJ, Willemze R, Starckx S, Opdenakker G, Fibbe WE. Neutrophils are indispensable for hematopoietic stem cell mobilization induced by interleukin-8 in mice. Proc Natl Acad Sci U S A 2002;99(9):6228-33.

40. Kobayashi SD, Voyich JM, Burlak C, DeLeo FR. Neutrophils in the innate immune response. Archivum Immunologiae et Therapiae Experimentalis 2005;53(6):505-517.

41. Kolaczkowska E, Kubes P. Neutrophil recruitment and function in health and inflammation. Nat Rev Immunol 2013;13(3):159-75.

42. Mayadas TN, Cullere X, Lowell CA. The multifaceted functions of neutrophils. Annu Rev Pathol 2014;9:181-218.

43. Lee $A$, Whyte MK, Haslett $C$. Inhibition of apoptosis and prolongation of neutrophil functional longevity by inflammatory mediators. J Leukoc Biol 1993;54(4):283-8.

44. May T, Wirth D, Hauser H, Mueller PP. Transcriptionally regulated immortalization overcomes side effects of temperature-sensitive SV40 large T antigen. Biochem Biophys Res Commun 2005;327(3):734-41.

45. Geng S, Matsushima H, Okamoto T, Yao Y, Lu R, Page K, Blumenthal RM, Ward NL, Miyazaki T, Takashima A. Emergence, origin, and function of neutrophil-dendritic cell hybrids in experimentally induced inflammatory lesions in mice. Blood 2013;121(10):1690-1700. 


\section{Figure legends}

Figure 1. Purity, survival and stimulation of bone marrow cell fractions. Bone marrow cells were isolated from mice expressing luciferase from an $\mathrm{Mx} 2$ promoter construct and fractionated into neutrophil-specific and monocyte-specific populations by using antibodycoupled magnetic beads (MACS). Ly6G and Ly6C antigen expression on the purified neutrophil bone marrow fraction (A) and on the monocyte fraction (B) was analyzed by FACS. Cultured cells isolated from the bone marrow of luciferase-expressing mice (C), a neutrophil-specific bone marrow fraction (D) and a monocyte-specific fraction (E) were stained using the intracellular esterase-activated fluorescent dye Calcein AM. The fluorescence intensity (RFU) was measured in plate reader with excitation and emission filters of $485 \mathrm{~nm}$ and $535 \mathrm{~nm}$, respectively. Symbols are as follows: Circles, heat inactivated bacteria were added to the culture; Triangles, control culture without bacteria. Dashed lines, luciferase activity (relative luminescence units, RLU); solid lines, relative fluorescence intensity (RFU).

Figure 2. Subcutaneously injected monocyte-specific bone marrow cells show persistent localized luciferase activity that can be stimulated by bacteria. $10^{5}$ luciferase-expressing total bone marrow cells $(\mathrm{BM})$, neutrophil-specific antigen expressing bone marrow cells $(\mathrm{N} \phi)$ and monocyte-specific bone marrow cells of luciferase-expressing mice $(M \phi)$, respectively, were subcutaneously injected with $(+)$ or without heat inactivated $S$. aureus bacteria (-). The luminosity was monitored by using an IVIS200 imager (A). The light emission from each injection site quantified individually at each time-point indicated in the presence of bacteria (B) or in the absence thereof (C). Error bars represent the standard deviation from 4 independent assays $(n=4)$. Symbols: Circles, total bone marrow cells; squares, neutrophilspecific bone marrow cell fraction; triangles, monocyte bone marrow cell fraction. 
Figure 3. Detection of bacterially contaminated implants after intravenous injection of luminescent bone marrow cells: $10^{6}$ total bone marrow cells $(\mathrm{BM})$, neutrophils $(\mathrm{Np})$ or monocytes $(\mathrm{Mp})$ isolated from the bone marrow of luciferase-expressing mice, were injected into the tail vein of mice carrying porous glass bead implants (1) or into a control animal without any surgical manipulation (2) and the resulting luminescence was monitored (A). White dashed circles indicate the location of implants; implants were as follows: b, porous glass bead coated with heat inactivated $S$. aureus bacteria; s, sterile porous glass bead implant; $i$, transcutaneous incision wound (A). Quantification of the luminosity at individual implantation sites after intravenous injection of total bone marrow cells (B), after injection of neutrophil-specific bone marrow cells (C) and after injection of monocyte-specific bone marrow cells (D). Each data point represents the average light intensity from individual implantation sites of four animals $(n=4)$. Error bars represent the standard deviation. Symbols are as follows: circles, implant with heat inactivated bacteria; squares, sterile implant.

Figure 4. Implant material-specific inflammation detected by intravenously injected bioluminescent bone marrow cells. PLGA coated titanium discs, plain titanium discs and magnesium discs were subcutaneously implanted in mice. Subsequently, luminescent bone marrow cells were injected into the tail vein and the luminosity was depicted (A) and quantified (B). PL: location of implanted PLGA coated titanium discs; Ti, titanium discs; Mg, magnesium discs (A). Circles, luminosity detected from PLGA coated titanium discs; squares, from magnesium implants; triangles, from titanium implants (B).

Figure 5. Differential responses of bone marrow cells to stable and biodegradable microparticle suspensions detected by in vivo imaging. PLGA and polystyrene microsphere solution and dissolved chitosan were injected subcutaneously in the mice. Luminescent bone marrow cells were intravenously injected and bioluminescent images were acquired (A) and the luminosity quantified (B) Dashed black circles indicate the site of injection. 1, Site of 
subcutaneous injection of a chitosan solution; 2, PBS buffer; $3,1 \mu \mathrm{m}$ diameter PLGA microsphere suspension; 4, $1 \mu \mathrm{m}$ diameter polystyrene microsphere suspension. Symbols are as follows: circles, polystyrene implants; asterisk, chitosan solution; triangles, PLGA implants; squares, phosphate buffered saline solution (PBS).

Figure 6. Histological examination of transient inflammatory responses to degradable PLGA and persistent response to polystyrene. PBS buffer (A), PLGA microbead suspensions (B) or polystyrene microbead suspensions (C) were subcutaneously injected and, after the time period indicated, tissue samples from the site of injection were fixed, paraffin embedded and stained. Headings: $\mathrm{H}$, microscopic overview of tissue thin sections stained with hematoxylin and eosin, black frames indicate the area depicted magnified on the right side; HE, enlarged image; MP, tissue sections stained with hematoxylin, eosin and antimyeloperoxidase antibodies; $\mathrm{d} 2, \mathrm{~d} 5, \mathrm{~d} 8$, tissue sample isolated 2 days, 5 days or 8 days after implantation, respectively. Black arrows point to a fibrotic tissue layer; white arrows indicate regions positively stained for myeloperoxidase activity. The scale bars correspond to $50 \mu \mathrm{m}$ (H) and to $100 \mu \mathrm{m}$ (HE and MP), respectively. Abbreviations: i, area containing the injected material; e, epidermis; d, dermis; ad, adipose tissue layer; sm, skeletal muscle; ct, connective tissue. 